\title{
Influence of dietary energy intake on nephrolithiasis - A meta-analysis of observational studies
}

\author{
Gianpaolo Perletti ${ }^{1,2}$, Vittorio Magri ${ }^{3}$, Pietro Manuel Ferraro ${ }^{4,5}$, Emanuele Montanari ${ }^{6}$, \\ Alberto Trinchieri ${ }^{7}$ \\ ${ }^{1}$ Department of Biotechnology and Life Sciences, Section of Medical and Surgical Sciences, University of Insubria, Varese, Italy; \\ ${ }^{2}$ Faculty of Medicine and Medical Sciences, Ghent University, Belgium; \\ ${ }^{3}$ Urology Secondary Care Clinic, ASST-Nord, Milan, Italy; \\ ${ }^{4}$ U.O.C. Nefrologia, Fondazione Policlinico Universitario A. Gemelli IRCCS, Roma, Italy; \\ ${ }^{5}$ Università Cattolica del Sacro Cuore, Roma, Italy; \\ ${ }^{6}$ Department of Urology, IRCCS Ca' Granda Ospedale Maggiore Policlinico - University of Milan, Milan, Italy; \\ 7 School of Urology, University of Milan, Milan, Italy.
}

\begin{abstract}
Summary Objective: Obesity has been associated with an increased risk of kidney stone formation. The presence of obesity is due to an imbalance between energy intake and energy consumption resulting from physical activity and resting metabolic rate. The purpose of this meta-analysis was to assess the differences in dietary energy intake levels between patients developing urinary stones versus healthy individuals.

Materials and methods: Medline/PubMed and EMBASE databases search was performed using the terms "urolithiasis", "kidney stones"”, "calcul*", "energy", "calor", "intake", "food", "kilojoule/kjoule", "Kilocal*/kcal" from January $1^{\text {st }}, 2000$, and were assessed as up to date on September 30 $0^{\text {th }}, 2019$.

Results: After having screened 1.782 records, four studies were included in the meta-analysis. The total population was 467.063, including 453.078 healthy men and/or women and 13.985 men and/or women affected by nephrolithiasis. When energy intake data were pooled irrespective of the sex of participants, mean calory intake values were significantly higher in nephrolithiasis patients, compared to healthy individuals. The mean difference (MD) was $39.16 \mathrm{kcal}$ (95\% CI 18.53 to $59.78, p=0.0002$, random-effects model, inverse-variance weighing). The odds ratio for this comparison - calculated from the standardized mean difference - is significant $(\mathrm{OR}=1.946$; 95\% CI: 1.869 to 5.561 ).

Conclusions: Patients affected by urolithiasis show a significantly higher energy intake in various patient populations (USA, China and Korea) including subjects of both sexes.

The relevance of this finding should be confirmed by studies in populations showing different and diverse dietary patterns, and by evaluating energy consumption linked to physical activity and metabolic rate in renal stone formers.
\end{abstract}

KEY WORDS: Urinary calculi; Energy intake; Obesity; Nutrition; Diet.

Submitted 14 October 2019; Accepted 29 February 2020

\section{INTRODUCTION}

A recent meta-analysis (1) confirmed that obesity is associated with an increased risk of renal stone formation. However, it has not been clarified which of the factors determining obesity are directly implicated in increasing the risk of formation of kidney stones.

In fact, obesity is due to the altered balance between dietary energy intake and energy consumption resulting from physical activity and resting metabolic rate.

The meta-analysis by Aune et al. (1) showed that renal stone formers do not have a lower level of physical activity when compared to non-stone forming subjects, but the impact of dietary energy intake on the risk of stone formation was not assessed in that study. The purpose of this meta-analysis was to investigate whether there were any differences between dietary energy intake levels in patients affected by urolithiasis versus healthy individuals, thus pointing to a possible association between dietary energy intake and the risk of renal stone formation.

\section{Materials AND MEthOdS}

Types of studies

We included only full-text articles written in English, reporting prospective cohort studies evaluating the relationship between daily dietary energy intake and the incidence of kidney stones in subjects of any age, men and/or women. We excluded case-control studies.

\section{Primary outcomes}

The single outcome considered for this review is the assessment of differences in dietary energy intake (mean daily kilocalories intake) between subjects developing kidney stones versus healthy individuals.

\section{Search strategy and study selection}

Records were identified by searching the Medline/PubMed, Cochrane and EMBASE databases, using the terms urolithiasis, kidney stone*, calcul*, energy, calor*, intake, food, kilojoule/kjoule Kilocal*/kcal. Hand searching was performed by browsing the references of published papers on the matter. Database searches were performed starting from January $1^{\text {st }}, 2000$, and were assessed as up to date on December $31^{\text {st }}, 2019$. This time frame has been chosen according to the meta-analysis of Aune et al. who selected studies published after 2000 .

Data extraction and analysis

Data extraction was performed by two independent 
researchers (GP, AT). When energy intake data in women (W) and men (M) were presented separately (e.g. the Shu 2017 study) (2) the combined mean energy intake and combined variance of the entire population (women and men) were calculated with the formulas: $\mathrm{X}=$ $(n W X W+n M X M) /(n W+n M)$ and $S=n W S W=$ $n M S M+N W(X W-X)^{\wedge} 2+n M(X M-X) \wedge 2 /(n W+n M) \quad(n=$ number of observations, $\mathrm{X}=$ mean, and $\mathrm{S}=$ variance). When energy intake data were expressed as median Kcal intake and interquartile ranges (IQRs) (e.g., the Kim 2018 study) (3), data were converted into means and standard deviations according to Wan et al. (4).

\section{Differences in energy intake between nephrolithiasis patients and healthy subjects}

To analyze differences in continuous data from the included studies (average daily energy intake, expressed as kilocalories/day) we calculated crude mean differences (MD) and standardized mean differences (SMD). Odds ratios for the comparisons were calculated with the formula: $\mathrm{OR}=$ antilnSMD* $\pi / \sqrt{ } 3$.

Analysis included the calculation of 95\% confidence intervals (CI) and Z statistics. For meta-analysis we adopted a random-effects model and the inverse variance weighing method. Heterogeneity was assessed by calculating the I^2 value. Pooled analysis was performed using the RevMan 5.3 software.

Funnel plots, the Egger's and Begg's tests, and "trim and fill" effect size adjustments were performed with the Meta-Essentials Excel workbook 1.0 (Erasmus Research Institute of Management, Erasmus University, Rotterdam, The Netherlands). Optimal information size for metaanalysis was calculated with the $C^{*}$ Power 3.1 software (assuming as an $\alpha$ level equal to 0.05 and $1-\beta$ error equal to 0.95 ).

\section{Assessment of risk of bias in included studies}

\section{Quality assessment}

The risk of bias (ROB) of included studies was assessed independently by two researchers (GP, AT).

This meta-analysis included non-randomized studies, and the Cochrane ROB tool had limited application.

We identified two main possible biases for the included studies: recall bias and reporting bias. Since recall and reporting biases may overlap to some extent, we adopted the following definitions: In the included studies, recall bias may have occurred when study participants forgot to recall past episodes of kidney stones (or the number of such episodes) during follow-up visits, whereas reporting bias may have occurred when patients gave inaccurate account of their food and energy intake by selectively revealing or omitting essential information.
The risk of bias was classified as low, high or unknown according to Cochrane criteria (5). Publication bias and small-study effect were investigated by visually assessing funnel plots and by performing both the Egger's regression test and the Begg's rank correlation analysis.

The "trim and fill" missing study imputation approach was applied to funnel plots and adjusted overall effect sizes were calculated according to Duval and Tweedie (6). The quality of evidence resulting from analysis of pooled data was evaluated according to GRADE criteria (7).

\section{Results}

\section{Description of studies}

After having screened 1.782 records, four studies (2, 3, 8, 9) including data from 6 cohorts were included in the meta-analysis (Table 1). The total population was 467.063, including 453.078 healthy men and/or women and 13.985 men and/or women affected by nephrolithiasis. From the studies by Shu et al. (2) and Sorensen et al. (8) the data regarding women only could be extracted (total: 153.391; healthy 149.548; nephrolithiasis: 3.843). Men strata could be extracted only from the Shu study (2) (total: 58.054; healthy 56.852; nephrolithiasis: 1.202).

\section{Meta-analysis}

When energy intake data were pooled irrespective of the sex of participants (men and women, or women only), mean energy intake values were significantly higher in urolithiasis patients, compared to healthy individuals. The mean difference (MD) was 39.16 calories (95\% CI: 18.53 to $59.78, \mathrm{p}=0.0002$; Figure 1). This comparison showed "considerable" heterogeneity ( $\left.\mathrm{I}^{\wedge} 2=79 \% ; \mathrm{p}=0.002\right)$.

The study of Ferraro et al. (9) was identified as a source of heterogeneity. When this study was excluded from analysis, the overall effect remained significant (MD: 49.57; 95\% CI: 37.73 to $61.40, \mathrm{p}<0.00001$ ) and the heterogeneity was eliminated $\left(I^{\wedge} 2=0, p=0.37\right)$. Sensitivity analysis was completed by excluding one by one the remaining studies, and the result remained significant (not shown).

Since the methods used to measure energy intake may have differed among studies, we confirmed the results of this analysis by calculating the standardized mean difference for the same comparison. The standardized mean difference was found to be equally significant (SMD: 0.07 units of standard deviation; 95\% CI: 0.03 to $0.12, \mathrm{p}=$ $0.001)$. The approximate odds ratio for the comparison is also significant (OR $=1.946$; 95\% CI: 1.869 to 5.561). Pooled comparison in women $(2,8)$ was devoid of statistical significance (MD: 21.41; 95\% CI: -23.95 to $66.78, \mathrm{P}=0.35$, Figure 1 ). Being based on only two: studies, this analysis does not allow further investigation.

\section{Table 1.}

Characteristics of included studies.

\begin{tabular}{|lll|}
\hline Study reference, location & Study design & Patients \\
\hline Ferraro 2015, USA & Prospective cohort study (20 years follow up) & Men and women; 25-75 years; Healthy: 209778; Nephrolithiasis: 5355: Total: 215133 \\
\hline Kim 2018, Korea & Prospective study (2011-2014) & Men and women; Healthy: 59728; Nephrolithiasis: 2363: Total: 62091 \\
\hline Shu 2017, China & Prospective cohort study (1996-2006) & Men and women; 40-70 years; Healthy: 124567; Nephrolithiasis: 2653: Total: 127220 \\
\hline Sorensen 2014, USA & Longitudinal prospective cohort study (1993-2006) & Postmenopausal women; < 50 years; Healthy: 81833; Nephrolithiasis: 2392: Total: 84255 \\
\hline TOTAL & & Healthy: 475906; Nephrolithiasis: 12763: Total: 488669 \\
\hline
\end{tabular}


Figure 1.

Pooled analysis of energy intake values in urolithiasis patients compared to healthy individuals.

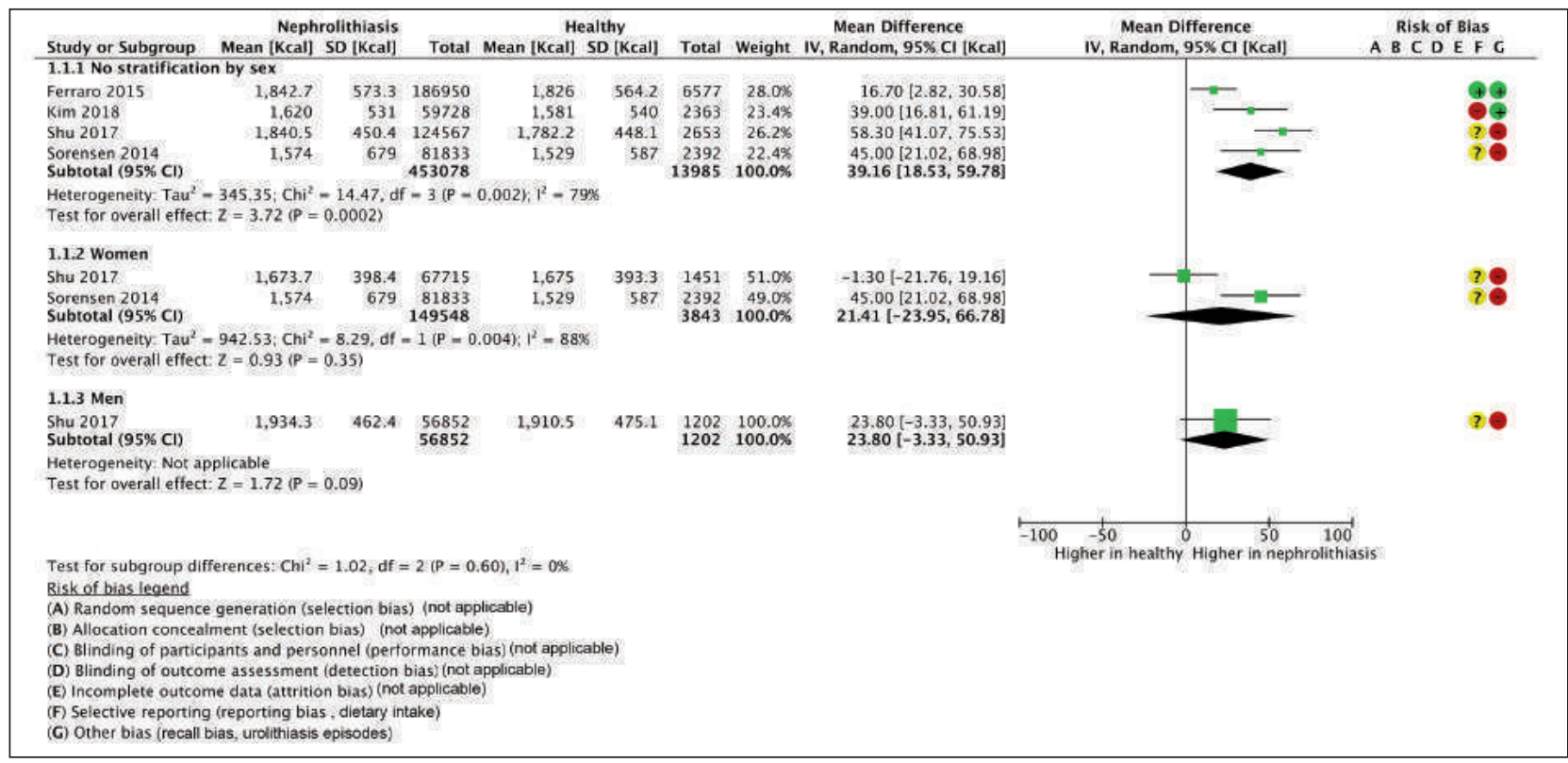

\section{Recall bias}

The recall in bias was rated as high in the Sorensen et al. (8) and Shu et al. (2) studies, as in both cases nephrolithiasis episodes were self-reported and not confirmed using a validated method. In the Kim et al. study (3) the risk of recall bias was low, since measures were taken (periodical ultrasonography) to confirm the self-reported occurrence of nephrolithiasis, as well to detect asymptomatic cases.

The recall bias was rated as low in the Ferraro et al. study (9), as self-reported episodes of kidney stones were confirmed by official medical records in over $97 \%$ of patients.

\section{Reporting bias}

The risk of reporting bias was reputed to be low in the Ferraro et al. study (9), as validated questionnaires were periodically administered to participants, and their analysis took into consideration corrections for energy-adjusted nutrients. In the Sorensen et al. study (8), attempts were made to correct some of the biases associated with reporting, as for energy intake was concerned. However, the authors admit that self-reported dietary intake in their study might have been prone to bias. Thus, the bias was rated as unknown. A validated food intake questionnaire was also administered by Shu et al. (2), but the Authors do not disclose whether any bias correction was attempted (unknown ROB). In the Kim et al. study report (3), the Authors did not disclose whether validated systems were implemented to assess the energy intake of patients (high risk of reporting bias). In the methods section, Kim et al. (3) refer to "standardized, self-administered questionnaires". However, such questionnaires assessed medical history, medication use, family history, physical activity, alcohol intake, smoking habits, and education level, but not dietary/energy intake.

\section{Publication bias}

Funnel plots were generated to analyze the publication bias for the comparison of data in the overall population (men and or women). The funnel plot indicated symmetry of the data distribution and such visual impression was confirmed by the Egger's test or by Begg's rank correlation analysis, as neither test reached statistical significance (Egger's, $\mathrm{p}=0.36$; Begg's, $\mathrm{p}=0.49$ ). The "trim and fill" method applied to the funnel plot imputed no missing studies (Figure 2), and no adjusted estimate of the overall effect size could be calculated for this comparison.

\section{Discussion}

The results of the present meta-analysis demonstrate a higher dietary energy intake in renal stone formers compared to non-stone forming subjects. This might be an important finding because it would better explain the association between obesity and the risk of renal stone formation

However, we still have limited data on the energy consumption in renal stone formers, which is linked to physical activity but especially to the resting metabolic rate, which is the largest component of daily energy consumption and is characterized by significant inter-individual differences (10). Furthermore, the energy intake of food nutrients that is calculated experimentally through direct calorimetry as thermal energy could differ from the net energy that is extracted through human metabolism. Efficiency of digestion for different foods depends on their coefficient of digestibility. Notably, energy loss occurs during the process of metabolism.

The present findings should be considered provisional and should be interpreted conservatively, since our meta-analysis was based on a limited number of observational studies from three countries (USA, China and Korea) that are characterized by very diverse dietary patterns. The typical US diet is based on abundant consumption of meat and animal fats, sugar, carbonated beverages, and insufficient consumption of fruits and vegetables. On the other hand, China and Korea are char- 
Figure 2.
The "trim and fill" method applied to the funnel plot imputed no missing studies.

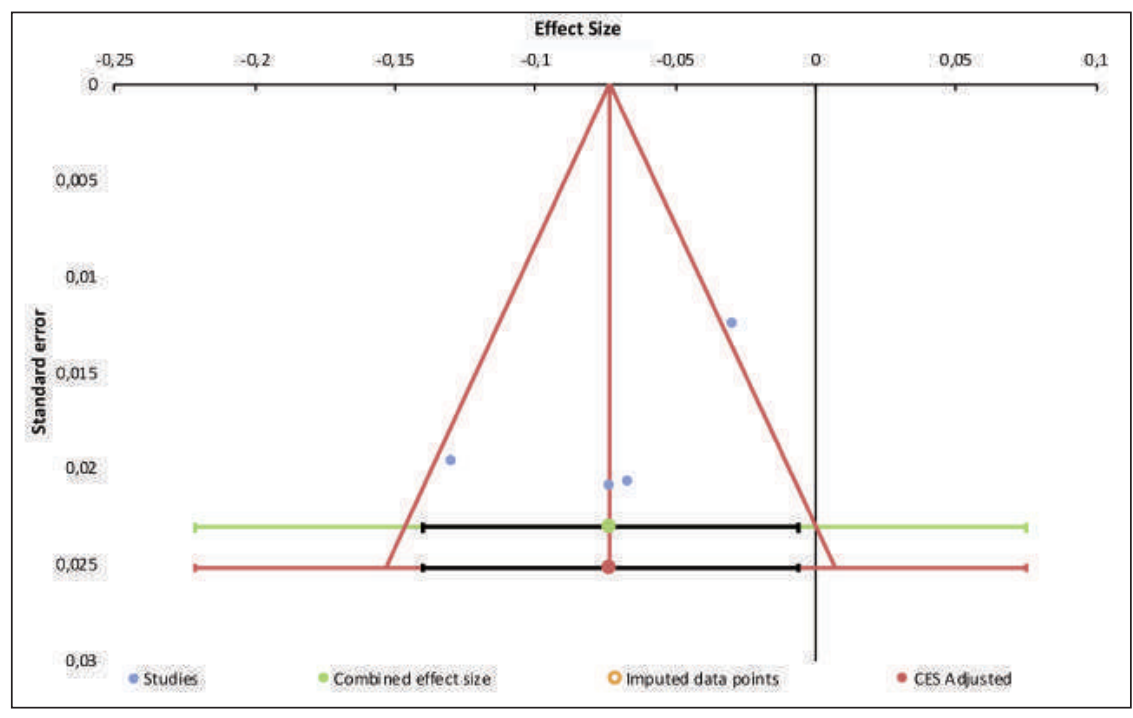

and meta-analysis of cohort studies. Eur J Epidemiol. 2018; 33:1033-1047.

2. Shu X, Cai H, Xiang YB, et al. Nephrolithiasis among middle aged and elderly urban Chinese: a report from prospective cohort studies in Shanghai. J Endourol. 2017; 31:1327-34.

3. Kim S, Chang Y, Sung E, et al. Association between sonographically diagnosed nephrolithiasis and subclinical coronary artery calcification in adults. Am J Kidney Dis. 2018; 71:35-41.

4. Wan $X$, Wang $W$, Liu J, Tong $T$. Estimating the sample mean and standard deviation from the sample size, median, range and/or interquartile range. BMC Med Res Methodol. 2014; 14:135.

5. Higgins JP, Altman DG, Gøtzsche PC, et al. Cochrane Bias Methods Group; Cochrane Statistical Methods Group. The Cochrane Collaboration's tool for assessing risk of bias in randomised trials. BMJ. 2011; 343: d5928.

acterized by dietary patterns that have a balanced intake of animal and plant-derived foods, at the same time based on a high sodium intake $(11,12)$. The association between dietary energy intake and the risk of renal stone formation could be different in other countries with healthier dietary patterns, with limited consumption of animal fats and meat and reduced salt consumption.

In particular, in countries consuming a Mediterranean diet, an increase in dietary energy intake could have a different effect on the risk of stone formation, as it is related to a higher intake of healthy foods such as cereals, nuts, fruit, vegetables, fish, olive oil, and dairy products. Hence, such food could cause an increase in body weight without concurrently increasing the risk of renal stone formation. In fact, it was shown that adherence to the Mediterranean diet (13) or the DASH diet (14), a diet suggested for the prevention of hypertension that provides for a reduction in the consumption of animal proteins and fats in favor of increased consumption of fruits and vegetables, is associated with a reduction in the risk of renal stone formation. In conclusion, the present meta-analysis, based on a large pool of subjects from the United States of America, China and Korea, showed that the mean energy intake of patients affected by urolithiasis is significantly higher than the corresponding intake in healthy individuals. Assessment of the standardized mean difference allowed the calculation of the odds ratio for such comparison. Such odds ratio (OR $=1.946$ (95\% CI: 1.869 to 5.561) is significant and points to an association between higher dietary energy intake and urolithiasis.

The extensive significance of this finding could be better evaluated when data from other populations with different dietary patterns and more data on energy consumption linked to physical activity and metabolic rate in renal stone formers will be collected.

\section{REFERENCES}

1. Aune D, Mahamat-Saleh Y, Norat T, Riboli E. Body fatness, diabetes, physical activity and risk of kidney stones: a systematic review
6. Duval S, Tweedie R. Trim and fill: a simple funnel-plot based method of testing and adjusting for publication bias in meta-analysis. Biometrics. 2000; 56:455-63.

7. https://training.cochrane.org/handbook

8. Sorensen MD, Chi T, Shara NM, et al. Activity, energy intake, obesity, and the risk of incident kidney stones in postmenopausal women: a report from the Women's Health Initiative. J Am Soc Nephrol. 2014; 25:362-9.

9. Ferraro PM, Curhan GC, Sorensen MD, et al. Physical activity, energy intake and the risk of incident kidney stones. J Urol. 2015;193:864-8.

10. Goran MI. Energy metabolism and obesity. Med Clin North Am. 2000; 84:347-62.

11. Zhang $R$, Wang $Z$, Fei $Y$, et al. The difference in nutrient intakes between Chinese and Mediterranean, Japanese and American diets. Nutrients. 2015; 7:4661-88.

12. Shu X, Calvert JK, Cai H, et al. Plant and animal protein intake and risk of incident kidney stones: results from the Shanghai men's and women's health studies. J Urol. 2019; 202:1217-1223.

13. Leone A, Fernández-Montero A, de la Fuente-Arrillaga C, et al. Adherence to the Mediterranean dietary pattern and incidence of nephrolithiasis in the Seguimiento Universidad de Navarra followup (SUN) cohort. Am J Kidney Dis. 2017; 70:778-786.

14. Taylor EN, Fung TT, Curhan GC. DASH-style diet associates with reduced risk for kidney stones. J Am Soc Nephrol. 2009; 20:2253-9.

\section{Correspondence}

Gianpaolo Perletti, Dr. Sci, M.Clin. Pharmacol. (Corresponding Author) gianpaolo.perletti@uninsubria.it

Department of Biotechnology and Life Sciences, Section of Medical and Surgical Sciences, University of Insubria, Varese, Italy

Vittorio Magri, MD - vittorio.magri@virgilio.it

Urology Secondary Care Clinic, ASST-Nord, Milan, Italy

Pietro Manuel Ferraro, MD - pietromanuel.ferraro@unicatt.it

U.O.C. Nefrologia, Fondazione Policlinico Universitario A. Gemelli IRCCS, Roma, Italy

Emanuele Montanari, MD - emanuele.montanari@unimi.it

Department of Urology, IRCCS Ca' Granda Ospedale Maggiore Policlinico -

University of Milan, Italy

Alberto Trinchieri, MD - alberto.trinchieri@gmail.com

School of Urology, University of Milan, Italy 